\title{
Entretien avec Mohamed Miloud Gharrafi
}

Interview with Mohamed Miloud Gharrafi

Mohamed Miloud Gharrafi

\section{CpenEdition}

Journals

Édition électronique

URL : http://journals.openedition.org/recherchestravaux/1812

ISSN : 1969-6434

Éditeur

UGA Éditions/Université Grenoble Alpes

Édition imprimée

ISBN : 978-2-37747-165-2

ISSN : 0151-1874

\section{Référence électronique}

Mohamed Miloud Gharrafi, «Entretien avec Mohamed Miloud Gharrafi », Recherches \& Travaux [En ligne], 95 | 2019, mis en ligne le 05 décembre 2019, consulté le 08 septembre 2020. URL : http:// journals.openedition.org/recherchestravaux/1812

Ce document a été généré automatiquement le 8 septembre 2020

(c) Recherches \& Travaux 


\section{Entretien avec Mohamed Miloud Gharrafi}

Interview with Mohamed Miloud Gharrafi

Mohamed Miloud Gharrafi

\section{NOTE DE L'ÉDITEUR}

Cet entretien a été élaboré à partir du matériau suivant : un entretien du 9 novembre 2017, mené par Camille Eynard, Gaëlle Grella, Gaëlle Le Port, Kevin Woringer, étudiants de Master (parcours «Littérature, critique et création ») à l'Université Grenoble Alpes, en ouverture de la première session du colloque «L'écrivain-traducteur : ethos et style d'un co-auteur "; un second entretien, le même jour, lors d'une lecture publique au Café des Arts animée par Pascale Roux. Ces deux entretiens, suivis ou précédés de lectures de textes, sont intégralement en ligne : <http://ouvroir-litt-arts.univgrenoble-alpes.fr/programmes/stylistique-du-texte-traduit/poetes-traducteurs-de-larabe>. Les textes ont été retranscrits et réorganisés par Pascale Roux, puis revus par Mohamed Miloud Gharrafi.

Question : Vous êtes l'auteur d'un certain nombre de livres en arabe ${ }^{1}$ et vous avez publié un livre bilingue, Échos lointains ${ }^{2}$, anthologie composée de textes déjà publiés et de textes inédits que vous avez vous-même traduits. Pouvez-vous nous parler de cette expérience de l'autotraduction?

Mohamed Miloud Gharrafi : L'autotraduction m'a été, au départ, imposée par les lectures publiques, où il fallait bien, après avoir lu le texte arabe, le faire passer en français. Comme je n'avais parfois pas le temps de demander à tel ou tel ami de traduire le texte, je m'y suis mis. C'est une expérience extraordinaire pour moi, une très très belle expérience. Elle est semée d'embûches, certes, mais l'autotraduction est une réécriture. Il m'est arrivé, alors que je traduisais certains textes, de me retrouver en train d'écrire un autre texte, pas complètement différent du texte arabe, mais tout de même un autre texte. La traduction est un mystère. En fait, on vous demande de traduire, de donner un équivalent de ce qui se trouve dans le texte 
d'origine mais, en poésie, cela n'arrive jamais, c'est une certitude. Un texte littéraire traduit ne peut jamais être le même que le texte de départ, ce n'est pas possible, parce que chaque langue a son propre fonctionnement syntaxique, sonore, que vous ne pouvez pas faire passer dans une autre langue, laquelle fonctionne autrement. Donc quand j'ai commencé à traduire certains de mes poèmes, il m'est arrivé de trouver le texte en français plus beau que le texte en arabe. Et alors je me suis dit: que se passe-t-il? je n'ai pas changé de sens, tout me semble être l'équivalent, si on peut dire, cela correspond à ce que je disais dans le texte en arabe, et pourtant ce n'est pas la même chose. Pourquoi certains textes que j'ai traduits en français me semblent-ils plus poétiques que les textes arabes et pourquoi d'autres le sont-ils moins? Et puis il y a une certaine insatisfaction dans ce genre de pratiques, parce qu'on a peur de porter atteinte au premier texte, au texte de départ. Je suis beaucoup plus à l'aise lorsqu'on me traduit dans des langues que j'ignore. On m'a traduit en espagnol deux fois, en italien, en macédonien, en roumain... Je me dis que cela ne me concerne pas. Le lecteur trouvera-t-il cela poétique ou non ? Je n'en sais rien, mais là, au moins, j'ai ce prétexte de ne pas savoir. Ce n'est certainement pas le cas lorsque vous traduisez vos propres poèmes.

Q: Vous êtes vous-même traducteur d'autres poètes ${ }^{3}$ : y a-t-il des points sur lesquels vous éprouvez que le passage de l'arabe au français pose des problèmes particuliers?

Mohamed Miloud Gharrafi : Un exemple de la difficulté de la traduction de l'arabe vers le français est la question du genre. En français, quand vous dites "Je t'aime ", c'est le contexte qui détermine si la personne à qui on s'adresse est un homme ou une femme. En arabe, le genre est déterminé dans la phrase elle-même : le pronom soudé à la fin de la forme verbale change selon que le destinataire est une femme ou un homme. On dit « ouhibbouki » pour une femme et «ouhibbouka » pour un homme. L'autre difficulté est d'ordre culturel. J'ai eu cette difficulté avec la traduction du recueil du poète égyptien Ahmed Al-Shahawi, Une seule porte et des demeures. Sa langue est on ne peut plus courante, moderne, elle n'appartient pas entièrement au registre de l'arabe classique, et pourtant les textes sont chargés de références culturelles, ils s'inspirent des grands poètes soufis (mystiques) comme Al-Hallaj, Ibn Al-Farid... Dans ce recueil, plusieurs titres sont tirés soit du texte coranique soit d'œuvres arabes littéraires ou mystiques classiques. Pour le lecteur arabophone, il suffit d'un peu de culture sur la poésie arabe classique, sur le texte coranique ou sur la poésie mystique arabe pour que le texte de référence revienne. Quand vous traduisez cela en français, le texte est accessible, mais, pour le lecteur francophone qui n'a pas une culture de cette langue-là, ce n'est pas le même texte que celui qui a été écrit en arabe. Le traducteur est donc obligé de faire des notes de bas de page pour le lecteur non arabophone.

Q : Pour ce qui concerne la forme poétique, quels sont vos partis-pris en arabe, sur la rime et le mètre, et quels choix de traduction avez-vous faits pour le texte français ?

Mohamed Miloud Gharrafi : Je ne partage pas l'avis de beaucoup de traducteurs de poésie selon lequel il faudrait aussi chercher à traduire la rime, pour la simple raison que, quand on cherche à traduire la rime, on peut exercer une certaine violence sur le texte. Chercher absolument à traduire la rime, c'est-à-dire à lui trouver un équivalent dans le texte d'arrivée, nous oblige dans la plupart des cas à nous éloigner du texte de départ. Donc quand je me suis traduit, je n'ai pas tenu compte de la rime, d'autant que je ne la pratique pas régulièrement dans mes poèmes. La rime y est à 
peine suggérée, elle n'est pas régulière ni systématique à chaque ligne, je laisse la rime venir toute seule quand il le faut. Quand elle arrive, je la saisis, mais je n'aime pas faire un poème en fonction d'une rime. J'ai pratiqué cela au début et, à un certain moment, c'est devenu monotone. D'autant plus que, dans l'histoire de la poésie arabe, celle-ci est restée, jusqu'aux années quarante, prisonnière de la rime, et non seulement de la rime, mais aussi de ce qu'on appelle le monorime, c'est-à-dire que tout le poème devait être construit sur des vers à deux hémistiches, le second se terminant toujours par la même rime. C'est la tradition poétique arabe, depuis la période préislamique, qui s'est imposée durant plusieurs siècles. Il a fallu attendre que les poètes arabes s'ouvrent sur la poésie occidentale, notamment anglaise et américaine, et française plus tard, pour qu'on commence à libérer le poème du monorime, et aussi de la métrique, parce que toute la poésie arabe classique est basée sur des mètres. Dans la théorie poétique arabe, qui date $\mathrm{du} \mathrm{Ix}^{\mathrm{e}}$ siècle, il existe 16 mètres. C'est dans ces mètres que les poètes arabes composaient. Dans les années quarante, un jeune poète, Badr Châkir al-Sayyâb, et une jeune poétesse, Nazik AlMalâ'ika - tous les deux irakiens - ont débarrassé la poésie arabe du monorime. Depuis cette période, les poètes arabes n'ont cessé d'expérimenter d'autres voies. On a toujours des poètes qui sont sur cette ligne du poème monorime, mais on les considère comme des poètes traditionnels, conservateurs pour ce qui concerne leurs choix esthétiques. Aujourd'hui, la poésie arabe comporte tout cela: le monorime, le poème avec des rimes alternées, et puis des poèmes sans rimes. Mais l'absence de rimes ne signifie pas l'absence de rythme, au contraire : lorsqu'on ne recourt pas à la rime, on essaie de jouer sur les sonorités, au niveau du choix des mots et de la syntaxe, on essaye de trouver pour le poème un certain rythme interne. Pour revenir au livre dont nous parlons, les poèmes français ne comportent pas de rime : c'est non seulement parce que je ne cherche pas absolument à traduire la rime, mais aussi parce que mes poèmes, à l'origine, notamment dans ce recueil-là, n'en comportent pas beaucoup.

\section{Q : La poésie pose donc des problèmes de traduction particuliers...}

Mohamed Miloud Gharrafi : C'est une expérience que je ne renouvellerai peut-être pas. S'autotraduire est une expérience difficile, il y a des poèmes auxquels je n'ai pas osé toucher, parce que, comme vous savez, le poème se définit par une certaine ambiguïté sémantique. Henri Meschonnic, à la fois grand traducteur et poète et qui a beaucoup travaillé sur la rime, dit qu'en poésie, le sens déborde : pas facile de mettre la main sur le sens pour le transmettre fidèlement dans une autre langue. Et quand on traduit, on traduit le sens, pas les mots. Il est facile de traduire un texte de presse, d'actualité, voire un texte de prose littéraire, tel une nouvelle ou un roman. Mais pour la poésie, c'est tout autre chose, parce que si on cherche à traduire le sens, on risque d'enlever au poème ce qui fait de lui un poème, c'est-à-dire son côté esthétique, sa littérarité. Il existe dans la traduction de la poésie arabe classique au moins trois tendances. La première cherche à traduire le sens et rien que le sens. La deuxième cherche à conserver les images, parce que c'est cela qui fait la force d'un poème lorsqu'on perd le rythme et la sonorité. La troisième cherche à traduire la rime aussi, à trouver une rime dans la langue d'arrivée. C'est pour cela que la traduction de la poésie pose problème. En réalité, si l'on cherche à traduire le sens, il faudrait d'abord pouvoir mettre la main sur ce sens, le saisir en intégralité, savoir exactement ce que le poète veut dire, pour pouvoir le transmettre dans une autre langue. Or, l'ambiguïté, le sens fugitif, sont des éléments nécessaires à la poésie. La 
traduction est un «acte de langage », disait Henri Meschonnic ${ }^{4}$. Et Maurice Blanchot écrivait: "Le sens du poème est inséparable de tous les mots, de tous les mouvements, de tous les accents du poème. Il n'existe que dans cet ensemble et il disparaît dès qu'on cherche à le séparer de cette forme qu'il a reçue. Ce que le poème signifie coïncide exactement avec ce qu'il est ${ }^{5}$.» En somme, toute traduction par la voie d'une interprétation du sens ou d'une explication paraphrasée est la négation même du poème. En poésie, nous dit Yves Bonnefoy, « Des significations, explicites ou implicites, voulues ou subies, simples ou complexes, on en trouve partout ", mais « elles y sont comme un empêchement à la poésie ${ }^{6}$ ». Il y avait pour moi certains poèmes qui me posaient problème au niveau du sens. Que voulais-je dire par là? me demandais-je. La question est en elle-même en contradiction avec une certaine conception de la poésie. Si je m'étais mis à interpréter ces poèmes, je serais rentré dans la traduction interprétative du "vers " ou plutôt de la ligne (puisque la notion de vers est quasiment absente de la poésie contemporaine). Faire ce choix-là aurait été risqué. J'ai donc choisi des poèmes qui se donnent à lire comme des nouvelles, des poèmes qui privilégient le récit, car ils posent moins de problèmes en traduction et je me suis dit qu'au moins, je pourrais rester fidèle à ce qu'il y a dans le texte en arabe.

Q: En plus des poèmes dans les deux langues, on trouve, dans Échos lointains, des calligraphies. Comment l'ouvrage a-t-il été conçu et quel lien existe-t-il entre ces calligraphies et les poèmes ?

Mohamed Miloud Gharrafi : Je n'ai jamais cherché à établir de lien entre le poème et la calligraphie dans ce recueil, puisque j'ai laissé le choix au calligraphe lui-même, Najeh Jegham ${ }^{7}$ : je lui ai soumis les textes et ne suis pas du tout intervenu, je lui ai laissé le choix d'être guidé par les poèmes et de faire des calligraphies, en fonction de la sensation, des émotions que les textes pouvaient provoquer en lui. Ce n'est donc pas moi qui ai cherché à établir un lien. Je vois bien que le calligraphe a parfois repris le titre du poème qu'il a calligraphié, mais la calligraphie ne se trouve pas toujours en vis-à-vis du poème dont elle est inspirée ; on la trouve parfois un peu plus loin, et là non plus, je ne suis pas intervenu. Le calligraphe est aussi l'éditeur du livre, et c'est lui qui a fait ces choix. Finalement, je deviens comme vous : un lecteur de ce recueil où je dois moi aussi chercher le lien entre le poème et la calligraphie. Là aussi, plusieurs lectures peuvent être faites de ce mariage entre le poème et l'art de la calligraphie. Nous sommes, dans ce livre, face à deux langages qui peuvent tantôt se croiser tantôt s'éloigner l'un de l'autre. Un choix éditorial et une caractéristique propres à L'aile éditions qui a publié ce recueil.

Q : Votre poésie est caractérisée par son insertion dans le monde moderne, par le biais des images que vous utilisez, des mots, qui sont issus de notre quotidien - par exemple la référence à Google, au monde numérique ou au téléphone portable. Pouvez-vous nous parler de ce choix?

Mohamed Miloud Gharrafi : C'est à dessein que j'utilise ce langage. Ma façon d'écrire mes textes est liée à une certaine tradition poétique arabe, basée sur le rythme - je n'ai pas coupé complètement avec l'héritage de la poésie arabe née au milieu du $\mathrm{xx}^{\mathrm{e}}$ siècle. Les deux poètes irakiens dont je parlais tout à l'heure ont appelé à une poésie dite « libre », « shi'r horr » (شعر حرّ), parce qu'ils pensaient qu'il fallait la libérer des règles et des contraintes de la métrique arabe classique, mais eux-mêmes ne se sont pas complètement libérés de cela, puisqu'ils ont gardé certains mètres, sur lesquels 
on compose jusqu'à présent. Le grand poète palestinien Mahmoud Darwich utilise dans sa poésie un langage issu de l'arabe moderne, mais tous ses poèmes sont composés sur des mètres qui ont été élaborés il y a longtemps. Choisir un langage quotidien, c'est donc aussi une façon de transgresser, d'aller chercher autre chose sans se couper totalement d'une certaine norme esthétique, tant que ce mélange est capable de créer quelque chose de nouveau en termes d'écriture. Vous me posez la question du langage que j'utilise : je le recherche volontairement parce que, tout en restant sur cette ligne du rythme, d'un certain mètre que j'emploie (mais pas d'une façon systématique), j'essaie aussi d'inscrire le poème dans ce qu'on appelle le poème en prose, c'est-à-dire un poème qui se nourrit de la banalité de notre vie de tous les jours, du quotidien. La poésie, c'est justement la capacité du poète de transformer le banal en œuvre d'art. Il y a beaucoup de choses que nous observons, qui nous entourent et que nous croisons ou utilisons tous les jours, des choses qui ne nous disent peut-être rien, mais en même temps beaucoup. Je saisis ces objets-là, ces moments qui semblent complètement banals, pour les intégrer dans le texte et les faire parler autrement.

Q: La traduction de ces éléments du monde moderne pose-t-elle des problèmes spécifiques? Les noms de marque, par exemple, ont-ils la même valeur culturelle dans deux langues comme l'arabe et le français?

Mohamed Miloud Gharrafi : Je fais référence à ces éléments parce qu'ils sont chargés de sens et me permettent d'arriver à ce qui fait la force d'un poème : la concision, c'est-à-dire exprimer beaucoup de choses en très peu de mots, suggérer. En poésie, il faut suggérer plutôt que dire explicitement la chose, et puis il faut respecter celui qui vous lit. Vous faites de lui un ignorant, un individu sans intelligence si vous lui donnez tout, si vous lui dites tout. Ce qui crée chez nous le plaisir de la lecture, et vous en avez sans doute fait vous-mêmes l'expérience, en lisant un roman, une nouvelle, un texte littéraire en général, un poème, est le fait de déceler par vousmêmes le sens ou l'image suggérés. Vous êtes presque submergés de joie lorsque vous comprenez par vous-mêmes quelque chose qui ne vous est pas donné d'emblée, qui vous était juste suggéré, et c'est cela qui vous donne le plaisir de poursuivre la lecture. Il suffit de dire "Google ", " Twitter ", " WhatsApp ", " Like », pour que cela déclenche en nous beaucoup de choses : les réseaux sociaux, les relations humaines et en même temps la solitude ; tout cela peut venir en une seule fois, dans un seul mot, c'est pour cela que ces mots sont importants. J'ai joué aussi sur des noms de marques (marques de vaisselle, de machines à laver...). Les images et le sens en poésie (si sens il y a) ne sont pas que le produit de la syntaxe. Il suffit d'un seul mot ou d'un nom propre pour déclencher l'image de tout un univers. Certains mots ont leurs équivalents en arabe tel que "bière " par exemple, mais le mot dialectal "birra " dans un poème, n'a pas tout à fait le même effet sur le lecteur que le mot (بيرّة) «jouaa » (جعة) qui désigne la même boisson en arabe classique. Le mot «birra » est dialectal, connu de tous et convoque à lui seul un ensemble de choses ancrées dans l'imaginaire du lecteur. Je travaille depuis un moment sur ce que la machine fait de l'homme, c'est important pour moi. Je puise donc beaucoup dans le langage moderne, technologique et vous avez peut-être remarqué, dans Échos lointains, quelques poèmes qui parlent du train, du voyage et des petites choses que l'on voit dans le train, qui peuvent avoir un sens particulier dans notre vie. 
Q : Vous parlez souvent du sens : le sens des choses, le sens en poésie... Vous est-il arrivé de trouver ou retrouver le sens d'un poème après l'avoir écrit?

Mohamed Miloud Gharrafi : Le but de la poésie n'est pas de transmettre un message. La poésie n'est pas un manifeste. Il y a d'autres discours pour véhiculer une idée ou un message précis. Ce qui fait l'âme de la poésie, comme je l'ai déjà dit, est la dimension fugitive du sens. Oui, il m'arrive parfois de m'interroger sur ce que je voulais exactement dire dans un poème, et tant mieux parce que c'est cette incertitude qui ouvre le poème sur l'inconnu, sur l'interprétation et lui donne, parmi tant d'autres éléments, sa part esthétique... Vous m'avez par exemple demandé de lire un poème ( «Le sable et $\left.\mathrm{moi}^{8} »\right)$ parce que vous l'aimez, mais moi je ne le considère pas comme un des meilleurs textes que j'aie écrits, sauf que ce texte-là, on me demande souvent de le lire. J'y parle d'une femme, j'évoque une autre femme, puis une troisième, et dans une succession d'images, je décris l'eau qui déborde et le sable saisi dans la main... Ce poème est ouvert à plusieurs interprétations... Je ne peux pas vous dire laquelle me conviendrait le mieux... Je ne me pose même pas la question de savoir s'il y en a une pour moi. Paradoxalement, ce texte a son succès auprès des lecteurs et je me demande encore si ce n'est pas cette ambiguïté et cette polysémie inhérentes à la poésie qui font son succès... Cela dit, la poésie n'est pas non plus un délire, un langage sans aucun sens. Il y a toujours un sens quelque part dans un poème, mais ce n'est pas toujours celui auquel pense le poète. Ce qui lie le poète au lecteur est tout d'abord un contrat esthétique.

Q: Votre poésie s'ancre dans la modernité : comment vous situez-vous par rapport aux traditions poétiques en langue arabe et en langue française?

Mohamed Miloud Gharrafi : Quand j'ai commencé à écrire, j'étais au collège. Et au collège, je n'avais pas beaucoup de choix puisque j'étais sous l'influence du modèle du poème monorime et monométrique qu'on étudiait en littérature arabe. Mes idoles étaient donc plutôt les grands poètes arabes classiques comme Tarafa, Al-Mutanabbi, Al-Maarri, Abou Tammam et bien d'autres. C'était ce modèle que j'essayais d'imiter tout au début de mon expérience poétique. Je l'ai exercé, pensant que c'était cela, la poésie. Et plus j'avançais, plus je découvrais qu'il y avait d'autres modèles. Et à un certain moment, vous savez, on se lasse, quand on fait toujours la même chose. En tout cas moi, je me lasse très rapidement. Je n'aime pas me répéter. Je vois autour de moi beaucoup de poètes qui écrivent sur un certain modèle depuis plus de 40 ans, je me pose parfois la question: comment peuvent-ils faire pour écrire toujours à l'intérieur du même schéma? Ils ont peut-être leur secret, ou bien c'est moi qui ne suis pas allé au plus profond des choses pour comprendre que, tout en gardant ce modèle-là, ils inventent autre chose. Je n'en sais rien. Ma première expérience a été donc le monorime. Après, quand j'ai découvert la poésie de Mahmoud Darwich, j'étais en troisième, je crois que c'est quelque chose qui m'a beaucoup bouleversé dans la conception que j'avais de la poésie. Mahmoud Darwich était le seul poète arabe qui pouvait remplir des stades en déclamant ses poèmes, et on était tous sous l'influence de sa poésie, qu'on appelait la poésie arabe moderne, qui était la suite de la poésie à laquelle avaient appelé ces deux grands poètes irakiens des années 40 dont je vous ai déjà parlé. Je l'ai exercée aussi, cette poésie-là, dite " libre ». Je continue à le faire. Mais je suis aussi passionné par le poème en prose, qui m'a ouvert d'autres horizons, qui m’a amené à réfléchir à la poésie comme quelque chose que l'on ne peut pas définir ou enfermer dans un modèle précis. Ce qui fait la force de la poésie est qu'elle reste ouverte à toute expérimentation. Aujourd'hui, dans le monde arabe, la plupart 
des jeunes poètes pratiquent ce qu'ils pensent être le poème en prose sans avoir suffisamment lu la poésie arabe classique et moderne. Pour écrire, il faut constamment baigner dans toutes ces expériences-là, du moins par la lecture... et pas que celle de la poésie. Un poème, ce n'est pas que le langage, ce ne sont pas que les mots, vous pouvez choisir, mettre autour de vous tous les mots du dictionnaire, les plus jolis que vous trouvez, mais cela ne fait pas un poème. Un poème se fait certes avec des mots, une syntaxe, mais aussi avec une certaine culture. La culture est importante dans l'écriture littéraire. On parlait tout à l'heure de ces objets de communication, d'Internet, de choses comme cela, qui appartiennent à la culture. Il faudrait donc puiser un peu, beaucoup, dans le passé et dans le présent à la fois, et dans d'autres cultures que la sienne: c'est cela, à mon avis, qui nourrit incontestablement l'écriture poétique. Pour revenir à votre question, je ne cherche pas à me situer dans un schéma ou un modèle précis. Quand j'écris je suis à l'écoute de moi-même, avec tout ce que ce « moi » rassemble en lui en termes de sentiments, d'expériences, de vécus, de lectures, mais je suis aussi à l'écoute des autres : textes, langues, œuvres d'art, expériences poétiques dans le monde arabe ou ailleurs. Pour écrire il faut mobiliser tous ces éléments-là. Il m'arrive d'écrire en français aussi avec le même esprit de liberté, d'écoute et de regard autour et à l'intérieur de moi.

\section{NOTES}

1. M. M. Gharrafi, حرائق العشق (Brûlures de la passion) : poèmes, Maroc, Tarifa, 2002 ; أمضغها علكات لم أر الشلالات من ; أسود (Ja la mâche comme un chewing-gum noir) : poèmes, Maroc, Tarifa, 2009 لا أشرك بالعزلة ; أعلى ) n'ai pas vu les cascades d'en haut) : roman, Beyrouth, Al-Ghawoon, 2011 أسون : أحدا (Je n'associe personne à ma solitude : poèmes), Le Caire, Dâr Al-Adham, 2014.

2. M. M. Gharrafi, Échos lointains, Nantes, L'aile éditions, 2014.

3. Euvres traduites par M. M. Gharrafi : A. Al-Shahawy, 2013, Une seule porte et des demeures, Nantes, L'aile éditions; A.Al-Hazmi, 2016, L'Arbre de l'absence, Nantes, L'aile éditions; M. A. Bennis, 2017, Regret en bas de la toile, Paris, L'Harmattan.

4. Voir notamment H. Meschonnic, 2007, Éthique et politique du traduire, Lagrasse, Verdier.

5. M. Blanchot, 1943, «La poésie de Mallarmé est-elle obscure?», Faux pas, Paris, Gallimard, p. 127-128.

6. Y. Bonnefoy, 2004, «La traduction de la poésie », Semicerchio : Rivisa di poesia comparata, $\mathrm{n}^{\circ} \mathrm{XXX}-\mathrm{XXXI}$, Florence, p. 68.

7. N. Jegham, présent dans le volume en tant que poète et que chercheur, est également calligraphe et éditeur (L'aile éditions, Nantes).

8. M. M. Gharrafi, « Le sable et moi », Échos lointains, ouvr. cit, p. 8. 


\section{RÉSUMÉS}

Mohamed Miloud Gharrafi, auteur de plusieurs recueils de poèmes en arabe, a signé, en 2014, une anthologie bilingue de ses poèmes autotraduits, augmentée d'inédits, intitulée Échos lointains. Il a accepté de s'entretenir avec nous sur sa pratique d'écrivain et de traducteur, et de présenter sa vision de l'acte de traduction et de ses relations avec la création, aussi bien dans le cas de l'autotraduction que dans celui de la traduction d'autres poètes, dont il a fait l'expérience à plusieurs reprises. Cette réflexion l'amène à poser la question des liens complexes, dans la poésie et sa traduction, entre la forme et le sens. Elle l'amène également à décrire son rapport, à la fois de filiation et de rupture, avec la tradition poétique arabe, ainsi que ses appartenances multiples à divers univers culturels et littéraires.

Mohamed Miloud Gharrafi, author of several collections of poems in Arabic, signed a bilingual anthology of his self-translated poems in 2014, supplemented by unpublished ones, entitled Échos lointains. He has agreed to talk with us about his practice as a writer and translator, and to present his vision of the act of translation and its relationship with creation, both in the case of self-translation and in the case of the translation of other poets, which he has experienced on several occasions. This reflection leads him to question the complex links, in poetry and its translation, between form and meaning. It also leads him to describe his relationship, both of filiation and rupture, with the Arab poetic tradition, as well as his multiple affiliations to various cultural and literary worlds. 